\title{
Philosophiques
}

\section{Roland Omnès, Philosophie de la science contemporaine , Paris, Folio Essais, Gallimard, 1994, 426 p.}

\section{Yvon Gauthier}

Volume 27, numéro 2, automne 2000

URI : https://id.erudit.org/iderudit/004927ar

DOI : https://doi.org/10.7202/004927ar

Aller au sommaire du numéro

Éditeur(s)

Société de philosophie du Québec

ISSN

0316-2923 (imprimé)

1492-1391 (numérique)

Découvrir la revue

Citer ce compte rendu

Gauthier, Y. (2000). Compte rendu de [Roland Omnès, Philosophie de la science contemporaine , Paris, Folio Essais, Gallimard, 1994, 426 p.] Philosophiques,

27(2), 460-462. https://doi.org/10.7202/004927ar d'utilisation que vous pouvez consulter en ligne.

https://apropos.erudit.org/fr/usagers/politique-dutilisation/ 
460 Philosophiques / Automne 2000

\section{Roland Omnès, Philosophie de la science contemporaine, Paris, Folio Essais, Gallimard, 1994, 426 p.}

Le physicien français Roland Omnès esquisse dans son essai une philosophie de la science contemporaine qui veut réhabiliter le sens commun afin de sortir de l'impasse où se trouve l'épistémologie contemporaine. C'est là le propos ou le voeu d'un auteur qui n'est ni philosophe des sciences, ni logicien mais qui semble croire qu'en ces matières tout honnête homme est pourvu de lumières naturelles et qu'il suffit en quelque sorte de réfléchir pour parvenir à des vues éclairantes sur toutes choses. Cette invitation à une philosophie populaire n'est en réalité qu'un travail de vulgarisation philosophique et on peut se demander si la philosophie spontanée du savant n'est autre chose que ce sens commun, si bien partagé entre les hommes que seuls les philosophes "professionnels» en seraient apparemment dépourvus.

La première partie de l'ouvrage porte sur « L'héritage » de la logique, de la physique, des mathématiques et de la philosophie de la connaissance classique ; on ne retiendra de la logique que le paradoxe du menteur d'Epiménide qui met en question le principe du tiers exclu (p. 52), pour la physique, on rappellera que le principe de moindre action (de Maupertuis repris par Lagrange) garde encore une grande part de son mystère (p. 90), pour les mathématiques classiques, on dira que Cantor a su se dépêtrer des paralogismes de la philosophie et de la théologie médiévales au sujet de l'infini (pp. 112-113) tout en négligeant de dire que Cantor s'est inspiré largement de l'une et de l'autre pour justifier sa théorie des ensembles transfinis ; pour la philosophie classique de la connaissance enfin, on concluera que les " méthodes transcendantales » chez Kant sont fondées sur la raison pure qui ne peut trancher les antinomies du fini et de l'infini (p. 131). Ce résumé est à peine caricatural ; en tout cas, il rend justice au genre, celui de l'épistémologue amateur qui se croit autorisé à débiter des généralités au nom du sens commun.

La deuxième partie de l'ouvrage porte sur « La rupture », sans doute épistémologique, entre l'héritage du passé et le partage du savoir actuel. On aura droit ici à d'autres paraphrases, par exemple sur le théorème de Gödel (pp. 175-178) dignes de figurer au palmarès de Sokal et Bricmont (eux aussi physiciens) :

L'exploit de Gödel fut de montrer qu'on peut effectivement assigner une valeur de vérité à certaines propositions sans avoir à passer par une démonstration, ce qui ne peut évidemment se faire qu'à l'aide d'une théorie de niveau supérieur dotée d'un métalangage. 
Un étudiant de logique du 1er cycle pourrait relever dans le dernier énoncé la confusion entre validité, théorème de consistance et notion de vérité ou encore le mélange entre le deuxième théorème d'incomplétude et le théorème de Tarski sur la vérité. Inutile de dire que l'éclairage sur la philosophie des mathématique dans ce contexte (chap. VI) est plutôt blafard et sagement l'auteur n'abordera pas l'intuitionnisme (ou le constructivisme), jugeant qu'il s'agit d'une matière trop technique (p. 192). Le chapitre suivant sur «La physique formelle " est une présentation générale de quelques concepts de la physique moderne. Le chapitre VII aborde "L'épistémologie de la physique " pour introduire une thématique chère à l'auteur, la théorie des histoires consistantes en mécanique quantique que nous voyons tout de suite.

La troisième partie de l'ouvrage est à cet effet consacrée au " Retour du formel au visuel » : il s'agit d'un exposé informel d'une interprétation non standard de la mécanique quantique dont l'auteur s'est fait le défenseur dans de nombreux travaux spécialisés parus surtout dans le Journal of Statistical Physics.

La théorie des histoires consistantes ou cohérentes (pour l'anglais « consistent histories ") remonte au physicien américain R.B. Griffiths qui a publié son article "Consistent Histories and the Interpretation of Quantum Mechanics » dans la revue mentionnée plus haut en 1984. L'interprétation de la MQ en termes d'histoires consistantes a été aussi adoptée par quelques physiciens importants dont Gell-Mann et Hartle. Disons brièvement de quoi il retourne : la théorie peut être considérée comme une variante de la théoire des multivers ( « many-universe interpretation ») d'Everett, mais avec une composante historique, puisque les mondes parallèles peuvent avoir des histoires différentes, i.e. des suites temporelles d'événements quantiques. Pour assurer qu'une histoire particulière est consistante, il faut lui accorder un statut logique affaibli qui lui interdit, par exemple, de conjuguer des événements incompatibles (e.g. les états de spin a et b d'un électron) dans une conjonction classique ab . Cette logique singulière d'une histoire quantique doit préserver les mesures de probabilité, ce que l'on appelle additivité sigma pour des mesures dénombrables. C'est ici qu'interviennent chez Griffiths et Omnès des notions de logique élémentaire et des considérations épistémologiques qui relèvent d'une introduction scolaire à la logique. Le modus ponens, les probabilités conditionnelles, les énoncés contrefactuels (dans la discussion entre Omnès et $\mathrm{B}$. D'espagnat citée en p. 311) y apparaissent comme des notions exotiques, comme si elles entraient en scène pour la première fois. Bien entendu toute la tradition épistémologique en philosophie de la physique y est ignorée au profit d'une sacralisation du sens commun qui aurait ainsi droit de cité dans les fondements mêmes de la mécanique quantique. Malheureusement, ce débat peu savant entre scientifiques sur les notions de vérité, de fiabilité (ou crédibilité, comme dit Omnès), de béance ou chiasme entre réalité empirique et réalité indépendante instruira peu le philosophe et risque d'égarer le scientifique. Il faut se demander si la théorie des histoires consistantes est consistante. Des travaux récents par Goldstein, Page, Dowker et Kent tendent à montrer que la théorie de Griffiths-Omnès est contradictoire - voir " On the Consistent Histories Approach to Quantum Mechanics " Journal of Statistical Physics, vol. 82, nos 5/6, 1996, pp. 1575-1646. La contradiction se situe au coeur même de la théorie, sur les mesures dans un espace de probabilité de cardinalité $2{ }^{\text {aleph }}$ - je note ici que j'avais indiqué la contradiction pour la théorie d'Everett dans mon article de 1983 "Quantum Mechanics and the Local Observer»Int. Journal of Theoretical Physics (vo. 22, no 12, 1983, pp. 1441-1152). D'un point de vue combinatoire, l'additivité dénombrable des probabilités suppose 


\section{Philosophiques / Automne 2000}

que la décomposition des mesures de probabilité recouvre aussi bien des sous-ensembles inconsistants que des sous-ensembles consistants irréconciliables dans la matrice de densité des histoires consistantes ; en d'autres termes, il n'y a pas de bijection entre $2{ }^{\text {aleph }}{ }_{0}$ et aleph ${ }_{0}$ et les probabilités standard ne sont plus récupérables dans des histoires proprement incohérentes. La morale de cette histoire, c'est que la logique interne des histoires consistantes est tout au plus une logique paraconsistante qui tolère la contradiction et s'accomode d'une " quasi-consistance " pour la quasi-classicalité, c'est-à-dire un mixte entre la cohérence des histoires dans les systèmes quantiques et leur décohérence dans les systèmes macroscopiques (classiques).

On retrouvera difficilement la trace de cette problématique dans la présentation informelle de l'auteur qui s'adonne à ce qu'on pourrait appeler une métaphysique expérimentale, si ce n'est à une physique spéculative. Il semble donc que le sens commun a sa dialectique propre qui n'ait rien à envier aux logiques non classiques.

Dans la quatrième et dernière partie de l'ouvrage "État des lieux et perspectives ", l'auteur dresse un bilan et esquisse quelques perspectives fuyantes. Entre l'image scientifique et l'image apparente du monde, selon l'expression de W. Sellars reprise par van Fraassen (The Scientific Image, Oxford, Oxford University Press, 1980), peut-on espérer que la philosophie (du sens commun ?) recommence (p. 398) ou renaisse dans la béance du visible physique et de son arrière-fond impré-visible (ou probabilitaire) ? Un glossaire à la fin de l'ouvrage explique en termes simples les concepts informels que l'auteur a essaimés sur son parcours réflexif.

On ne contestera pas la légitimité de l'entreprise et l'évaluer critiquement n'est pas un constat d'invalidité. Mais on reconnaît que la philosophie critique des sciences a une vocation fondationnelle qui diffère de la philosophie générale de la science dans la mesure où elle s'écarte résolument des thèses métaphysiques traditionnelles pour utiliser les ressources techniques et les moyens formels du savoir actuel. L'idée d'une logique et d'une épistémolo-logique interne du discours scientifique ne s'identifie pas à la philosophie "naturelle » du savant et s'il n'est pas interdit au praticien de produire la philosophie de sa pratique, on lui demande la même rigueur partout. L'ouvrage d'Omnès, après ceux de Prigogine et de nombreux autres, ne s'adresse sans doute pas aux philosophes des sciences (avertis!), mais les néophytes n'y trouveront pas nécessairement leur compte, malgré la clarté du style et la simplicité des idées que le scientifique a voulu rendre accessibles au commun des mortels. 